\title{
Increasing FTIR spectromicroscopy speed and resolution through compressive imaging
}

\author{
Julien Gallet ${ }^{\dagger}$, Michael Riley ${ }^{\ddagger}$, Zhao Hao and Michael C. Martin ${ }^{*}$ \\ Advanced Light Source Division, Lawrence Berkeley National Laboratory, 1 Cyclotron Road, Berkeley, CA 94720, USA \\ †Visiting from: Ecole Nationale Supérieure d'Ingénieurs de Caen, ENSICAEN, 6 bld du Maréchal Juin 14050 CAEN Cedex, France
}

${ }^{\ddagger}$ Permanent address: 309 Brook St. Westbrook, ME 04092

\begin{abstract}
At the Advanced Light Source at Lawrence Berkeley National Laboratory, we are investigating how to increase both the speed and resolution of synchrotron infrared imaging. Synchrotrons infrared beamlines have diffraction-limited spot sizes and high signal to noise, however spectral images must be obtained one point at a time and the spatial resolution is limited by the effects of diffraction. One technique to assist in speeding up spectral image acquisition is described here and uses compressive imaging algorithms. Compressive imaging can potentially attain resolutions higher than allowed by diffraction and/or can acquire spectral images without having to measure every spatial point individually thus increasing the speed of such maps. Here we present and discuss initial tests of compressive imaging techniques performed with ALS Beamline 1.4.3's Nic-Plan infrared microscope, Beamline 1.4.4 Continu $\mathrm{m}$ XL IR microscope, and also with a stand-alone Nicolet Nexus 470 FTIR spectrometer.
\end{abstract}

Keywords: Synchrotron; Spatial Resolution; Microscopy; FTIR; Spectromicroscopy; Imaging; Diffraction; Compressive Imaging; Hadamard

\section{Introduction}

Synchrotron infrared (IR) beamlines provide diffraction-limited spatial resolution for spectromicroscopy with $100-1000$ times higher brightness than a conventional thermal globar source [1-4] enabling a wide variety of new science at small spatial scales $[3,4]$. We have previously experimentally verified the diffraction-limited performance through the mid-IR at the Advanced Light Source (ALS) [5,6]. Our interest now is to see if one can do better: the two primary directions for improvement are higher spatial resolution (beyond the diffraction limit), and faster data acquisition.

Focal plane array infrared microscopy imaging systems are becoming more available and have the ability to acquire images significantly faster than point-by-point measurements, albeit at much lower signal to noise than a synchrotron source [5]. To achieve higher imaging speeds with a synchrotron source, one could spread the focus or rapidly raster a synchrotron beam onto a sub-set of pixels on a focal plane array and therefore give up some signal strength to image somewhat faster.

Alternatively we can explore if every pixel's measurement is really needed! Image compression algorithms have advanced in recent decades with

\footnotetext{
* Corresponding author. Tel.: +1-510-495-2231; fax: +1-510-495-2067; e-mail: MCMartin@lbl.gov.
} 
popular schemes such as JPEG in regular worldwide use to significantly compress image sizes. Compression algorithms remove redundant information from an image and one can trade-off image fidelity and resolution for file size.

Compressively sampled imaging uses spatial masks to sample various regions of the object, then selective measurements are combined to determine the intensity of each pixel and thus build up the image [7-10]. The more measurements acquired, the higher the quality and resolution the resultant image can become. This provides an important knob to choose between the quality of the image and the speed of acquisition. This technique can be used for spectral imaging by obtaining complete spectra for each mask, and then building up the spectral image using the same compressive imaging algorithms.

\section{Hadamard Masks}

Figure 1 shows the 64 spatial masks created using an $8 \times 8$ Hadamard sequence, and the corresponding Hadamard matrix. The masks progress to higher spatial frequency from the upper left to lower right.

Measurements of the intensity or spectrum of a sample are done through each mask starting with the

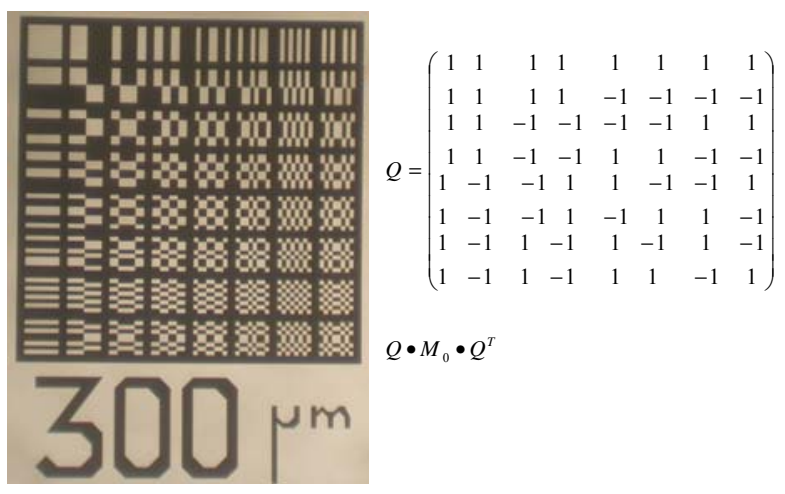

Fig 1. Micrograph of an 8x8 Hadamard mask with each mask's dimension being $300 \mu \mathrm{m}$. The corresponding Hadamard matrix Q is shown at the left, along with the applied matrix transformation of the measured intensity through each mask, $\mathrm{M}_{0}$.

upper left (largest opening) to the lower right (highest spatial frequencies). Linear additions of each of the
64 measurements will result in the unique intensity for each pixel in the real image. This can be accomplished using straightforward matrix algebra, with the primary conversion shown in Fig. 1.

Images can be reconstructed without measuring all 64 masks [7-10]. If one does not use the higher spatial frequency mask measurements, images will still be reconstructed with less spatial resolution. Indeed much like JPEG compression, reasonable images can be obtained using significantly fewer measurements than the number of pixels in the final image. In this paper we show initial tests we have performed reconstructing simple test images using more and less measurements using Hadamard masks.

\section{Experiment}

We performed several types of compressive spectral imaging test measurements using the FTIR microscopy equipment at ALS Beamline 1.4.3 and 1.4.4. We micro-fabricated Hadamard masks (Fig. 1 shows an example) as chromium on glass lithography masks. Some tests were preformed with the Hadamard masks located in the aperture plane before the sample in the IR microscope. In this case we mounted an automated $x-y$ stage to hold and scan through the various masks at the upper aperture of the Nic-Plan microscope. Other tests used transmissive or reflective Hadamard masks mounted on the main sample stage and test geometric shapes were made using the reflex aperture of the Continuum XL microscope. For every test sample, we collected the IR spectrum through each Hadamard mask, then reconstructed the images using matrix algebra via a Mathematica script. We reconstructed each images using progressively less of the measured data to explore how the number of measurements correlate with the resultant spatial resolution and image quality of the test sample.

Example reconstructions of a diagonal test illumination shape are shown in Fig. 2 using both $8 \times 8$ and 16x16 Hadamard masks. We see that using between 11 and $25 \%$ of the total masks (using the upper diagonal of the measured matrix) allow a reasonable reconstruction of the diagonal test object. 

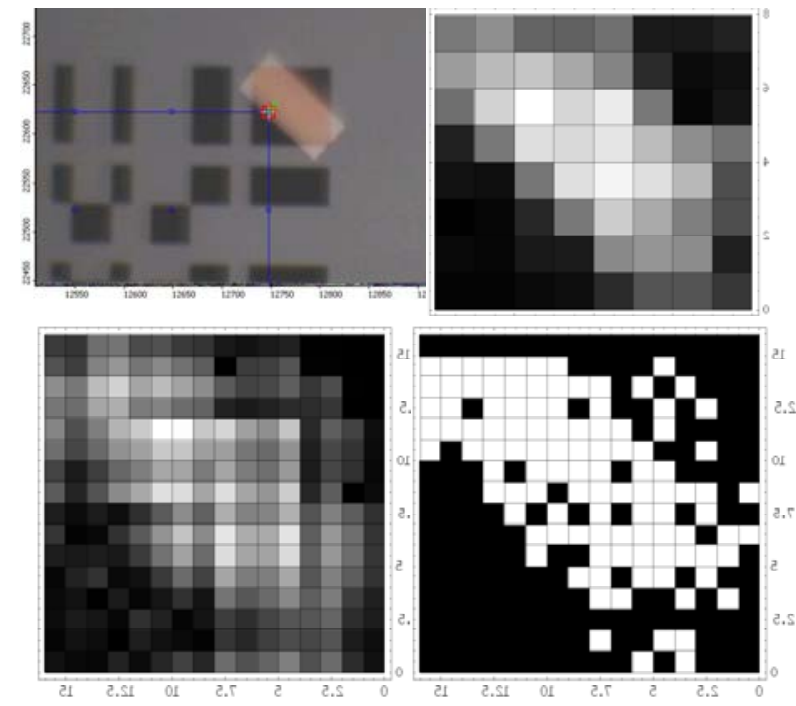

Fig 2. Example compressive imaging test. Upper left: micrograph of the test pattern showing this diagonal test illumination placed on the first Hadamard mask in reflection geometry. Upper right: reconstruction showing diagonal from the near-IR spectra acquired through $25 \%$ of the $8 \times 8$ Hadamard masks. Lower left: reconstruction using $11 \%$ of the $16 \times 16$ masks. Lower right: reconstruction using $82 \%$ of the $16 \times 16$ masks and enhanced contrast.

\section{Toward sub-diffraction limited imaging}

The above tests demonstrate the principle of using compressive imaging with spectromicroscopy. This is an intriguing tool that could enable users of the method to trade-off speed for imaging resolution. An exciting extension of this would be to start with the diffraction-limited synchrotron beam, and use a series of Hadamard masks having spatial features smaller than the wavelength to achieve images with subdiffraction-limited resolution. In this case the masks must be within the near-field of the sample under study otherwise diffraction would quickly smear out the resolution.

Using Hadamard masks in the near-field may have a significant advantage over many other highly lossy near-field probing techniques in that the masks all have $\sim 50 \%$ open area which should allow a huge amount more signal than when forcing light through a sub-wavelength aperture. Topographic artifacts may also be less important than with scanning probe techniques. We plan to continue testing compressive imaging with real samples and to demonstrate subwavelength compressive imaging. We also want to test other compressive algorithms that have been developed which use randomly generated masks.

\section{Acknowledgements}

This work and the Advanced Light Source are supported by the Director, Office of Science, Office of Basic Energy Sciences, Materials Sciences Division, of the U.S. Department of Energy under Contract No. DE-AC02-05CH11231 at Lawrence Berkeley National Laboratory.

\section{References}

[1] J. A. Reffner, P. A. Martoglio, and G. P. Williams, Rev. Sci. Instrum. 66, 1298 (1995); G. L. Carr, J. A. Reffner, and G. P. Williams, Rev. Sci. Instrum. 66, 1490 (1995) ; G. L. Carr, Rev. Sci. Instrum. 72, 1613 (2001).

[2] Michael C. Martin and Wayne R. McKinney, Proceed. Mater. Res. Soc., 524, 11 (1998); Michael C. Martin and Wayne R. McKinney, Ferroelectrics, 249(1-2), 1-10, (2001).

[3] Hoi-Ying N. Holman, Michael C. Martin, and Wayne R. McKinney, Spectroscopy - An International Journal 17(2-3), 139-159 (2003); Hoi-Ying N. Holman and Michael C. Martin, Advances in Agronomy 90, 79-127 (2006).

[4] Paul Dumas and Mark J. Tobin, Spectroscopy Europe 15 (6), 17-23 (2003).

[5] Erika Levenson, Philippe Lerch, and Michael C. Martin, Infrared Physics and Technology 49, 45-52 (2006).

[6] Erika Levenson, Philippe Lerch, and Michael C. Martin, Infrared Physics and Technology, this volume.

[7] N. P. Pitsianis, D. J. Brady and X. Sun, Proc. SPIE 5817, 250 (2005).

[8] D. J. Brady, M. Feldman, N. Pitsianis, J. P. Guo, A. Portnoy and M. Fiddy, Proc. SPIE 5907, (2005).

[9] Michael Wakin, Jason Laska, Marco Duarte, Dror Baron, Shriram Sarvotham, Dharmpal Takhar, Kevin Kelly, and Richard Baraniuk, Proc. International Conference on Image Processing -- ICIP 2006, Atlanta, GA, IEEE, (2006).

[10] Iren Valova and Yukio Kosugi, IEEE Trans. Inform. Tech. in Biomedicine, 4, 306-319 (2000). 